\title{
Correction to: Eye injuries from fireworks used during celebrations and associated vision loss: the international globe and adnexal trauma epidemiology study (IGATES)
}

\author{
Annette K. Hoskin ${ }^{1,2} \cdot$ Rebecca Low ${ }^{3} \cdot$ Jan Tjeerd de Faber ${ }^{4}$. Chitaranjan Mishra ${ }^{5}$. Pradeep Susvar ${ }^{6}$. Eli Pradhan ${ }^{7}$. \\ Andres Rousselot ${ }^{8}$. Fasika A. Woreta ${ }^{9} \cdot$ Lisa Keay $^{10,11}$. Stephanie L. Watson ${ }^{1} \cdot$ Rupesh Agrawal $^{3}$ (ID . \\ IGATES Fireworks study group
}

Published online: 13 September 2021

(c) Springer-Verlag GmbH Germany, part of Springer Nature 2021

\section{Correction to: Graefe's Archive for Clinical and Experimental Ophthalmology https://doi.org/10.1007/s00417-021-05284-z}

In the original published article, one of the "IGATES Fireworks study group" collaborators name has been misspelled.

"Rekha Khanelwal" should be "Rekha Khandelwal"

This is being corrected in this publication.

Publisher's note Springer Nature remains neutral with regard to jurisdictional claims in published maps and institutional affiliations.

The original article can be found online at https://doi.org/10.1007/ s00417-021-05284-z.

Rupesh Agrawal

Rupesh_agrawal@ttsh.com.sg

1 Save Sight Institute, Discipline of Ophthalmology, Sydney Medical School, The University of Sydney, Sydney, New South Wales, Australia

2 Lions Eye Institute, The University of Western Australia, Perth, Australia

3 National Healthcare Group Eye Institute at Tan Tock Seng Hospital, Singapore 308433, Singapore

4 The Rotterdam Eye Hospital, Rotterdam, Netherlands

5 Aravind Eye Hospital, Madurai, India
6 Sankara Nethralaya, Chennai, India

7 Tilganga Institute of Ophthalmology, Kathmandu, Nepal

8 Ophthalmology Department, Universidad del Salvador, Buenos Aires, Argentina

9 Wilmer Eye Institute, Johns Hopkins University School of Medicine, Baltimore, MD, USA

10 School of Optometry and Vision Science, UNSW, Sydney, Australia

11 The George Institute for Global Health, UNSW, Sydney, Australia 